\title{
Pilot study of the multifocal electroretinogram in ocular hypertension
}

\author{
Henry H L Chan, Brian Brown
}

\begin{abstract}
Aims-To investigate the variation of retinal function in ocular hypertension (OHT) using the multifocal electroretinogram (MERG), that can objectively assess regional retinal responses using kernel analysis.

Methods-Patients with OHT were recruited for 61 flash MERG recording. The first and second order kernel responses were analysed and compared with responses from a normal group. All the patients with OHT had full eye examinations and visual field analysis to ensure they had no ocular pathology, apart from high IOP ( $\geqslant 22 \mathrm{~mm} \mathrm{Hg}$ ).

Results-In OHT, both the first and the second order kernel responses showed a reduction in magnitude compared with control values. The second order kernel responses showed larger relative reduction of amplitude than the first order responses. In addition, the macula showed a greater reduction in response than the periphery.

Conclusions-First order and second order kernel analyses are useful for detecting changes of retinal response in OHT. The second order kernel analysis is important in measuring the inner retinal activity and is an important factor in detecting the early glaucoma case. Diminished macular response may be a useful sign in early glaucomatous changes. (BrF Ophthalmol 2000;84:1147-1153)
\end{abstract}

Right superior-

nasal (SN)

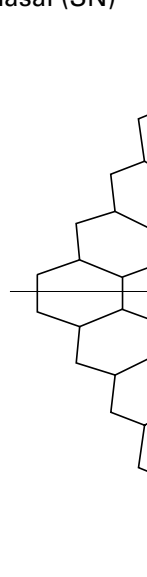

Right inferiornasal (IN)
Right superiortemporal (ST)
Department of Optometry and Radiography, Hong Kong Polytechnic University, Hong Kong H H L Chan

B Brown

Correspondence to: Dr Chan orhenry@polyu.edu.hk

Accepted for publication 26 April 2000
Glaucoma is a common eye disease that causes optic nerve fibre damage. It results in visual field constriction and loss of central vision. ${ }^{1}$ Elevated intraocular pressure (IOP) at the optic nerve head may cause tissue congestion at the lamina cribrosa and disrupt axoplasmic transport. ${ }^{2}$ This may be the cause of the damage in glaucoma. Measurement of IOP is thus important in the assessment of suspected glaucoma, but IOP shows considerable variation between patients. There is a problem in determining whether a patient has glaucoma if the IOP is in the marginal range (normal upper limit $\sim 21 \mathrm{~mm} \mathrm{Hg}$ ). ${ }^{3}$

Significant reductions of pattern electroretinogram (PERG) response have been reported in patients with ocular hypertension (OHT) and glaucoma. ${ }^{4-11}$ However, the PERG cannot provide a measure of pathological changes in different retinal areas. The multifocal electroretinogram (MERG) ${ }^{12}$ can examine the retina to give a clear indication of central and peripheral electrical responses. ${ }^{13}$ Localised retinal defects have been shown in retinitis pigmentosa, ${ }^{14}$ optic nerve atrophy, ${ }^{15}$ and retinal detachment. " "Kernel" analysis of MERG responses can be used to assess non-linear functions of the visual system. ${ }^{17}$ The "first order kernel" response (which reflects the average retinal response to a focal flash) and the "second order kernel" response (which reflects retinal temporal interactions-that is, fast adaptive processes) $)^{1518} 19$ in OHT and normal subjects were compared in this pilot study.

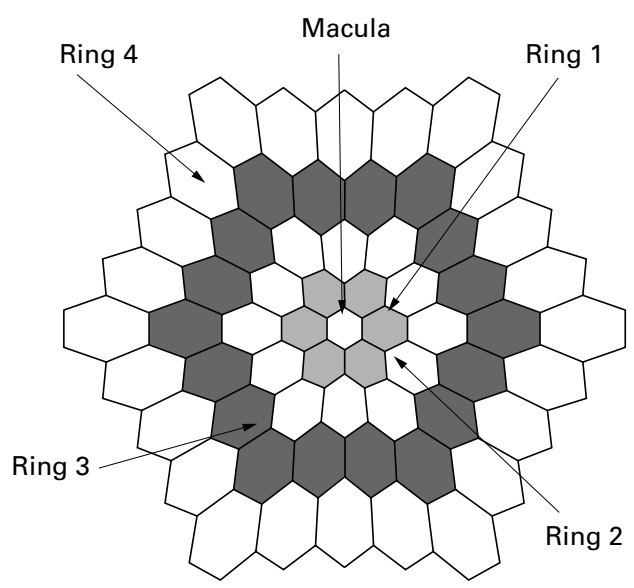

Figure 1 Schematic diagram of the 61 hexagon MERG stimulus pattern. The large cross at the centre of the pattern was used to divide the four quadrants of the MERG stimulus results of the right eye for analysis. The hexagons lying on the division line were not used for the analysis (left). The positions of the different regions (macula, rings 1-4) are shown in the stimulus pattern (right). 

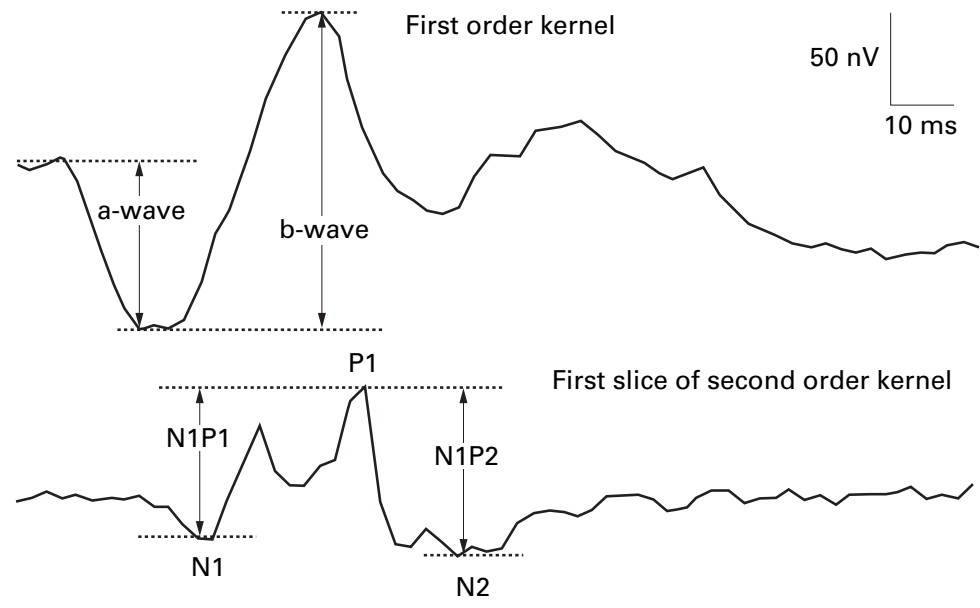

Figure 2 The waveforms and measurements of the first order kernel response (upper) and the first slice of the second order kernel response (lower) from the MERG.

\section{Methods}

SUBJECTS

Eight OHT subjects (five male and three female), ranging in age from 22 to 63 years (mean age 34.9 years), were recruited from the optometry clinic at the Hong Kong Polytechnic University. Twelve eyes in this group were tested in the study. The range of corrected visual acuity was from 0.0 to $0.08 \log \mathrm{MAR}$ (mean 0.04), and the range of cup/disc ratios

Table 1 Details of the ocular hypertension (OHT) subjects

\begin{tabular}{llllll}
\hline Subject & Tested eye & Age & VA & C/D & IOP \\
\hline MC & L & 22 & 0 & 0.6 & 23 \\
MC & R & 22 & 0 & 0.6 & 22 \\
LC & R & 22 & 0 & 0.5 & 23 \\
SML & R & 24 & 0.02 & 0.5 & 22 \\
SL & L & 25 & 0.06 & 0.2 & 23 \\
SM & L & 30 & 0.06 & 0.3 & 22 \\
SW & L & 31 & 0.04 & 0.45 & 26 \\
SW & R & 31 & 0.04 & 0.3 & 24 \\
YY & L & 43 & 0.04 & 0.7 & 23 \\
YY & R & 43 & 0.08 & 0.7 & 23 \\
PC & L & 63 & 0.06 & 0.5 & 22 \\
PC & R & 63 & 0.06 & 0.4 & 22 \\
\hline
\end{tabular}

All the OHT subjects have the open anterior angles (van Herick ratio $>0.7$ ). was from 0.2 to 0.7 (mean 0.48). The IOP measured by Goldmann tonometry ranged from 22 to $26 \mathrm{~mm} \mathrm{Hg}$ (mean $22.9 \mathrm{~mm} \mathrm{Hg}$ ). These IOP values were the mean of nine values. The ocular media were clear in all subjects. The anterior angles of the OHT subjects were examined using the van Herick test and gonioscopy. All patients had open angles (van Herick ratio $>0.7$ ) and no patient had plateau iris. Table 1 gives further details of the subjects. No subject had visual field losses when tested with the Humphrey II visual field analyser (30-2 threshold program). A normal visual field was determined using the STATPAC software provided with the Humphrey visual field analyser. (Visual fields were comparable in the two groups; the visual field mean deviation in the ocular hypertension group and the control group were 1.36 (2.56) $\mathrm{dB}$ and 2.05 (2.31) dB respectively. These values are not statistically different $(t=0.68, \mathrm{p}=0.49)$.)

There were 19 normal subjects (nine male and 10 female), ranging in age from 21 to 62 years (mean age 31.1 years). Nineteen eyes in the normal group were tested with MERG recording. All of these subjects passed tests to exclude the presence of glaucoma (criteria: negative family history, cup/disc ratio $(\leqslant 0.4)$, normal neural rim appearance, IOP $<21 \mathrm{~mm}$ $\mathrm{Hg}$ (measured by Goldmann tonometry), van Herick ratio $>0.7$, no plateau iris, clear ocular media, and no visual field defect with the Humphrey II visual field analyser (30-2 threshold program)). The range of corrected visual acuity of the normal subjects was from -0.1 to $0.06 \log$ MAR (mean -0.02 ).

Both OHT and normal subjects had refractive errors from plano to $-6.00 \mathrm{D}$ and less than $-2.00 \mathrm{D}$ astigmatism. There were no significant differences in the eye examination results other than IOP between the two groups.

Informed consent was obtained from all subjects after the experiments were explained.

Table 2 Statistical results of the MERG in OHT and control groups in different groupings (five ring and four quadrant)

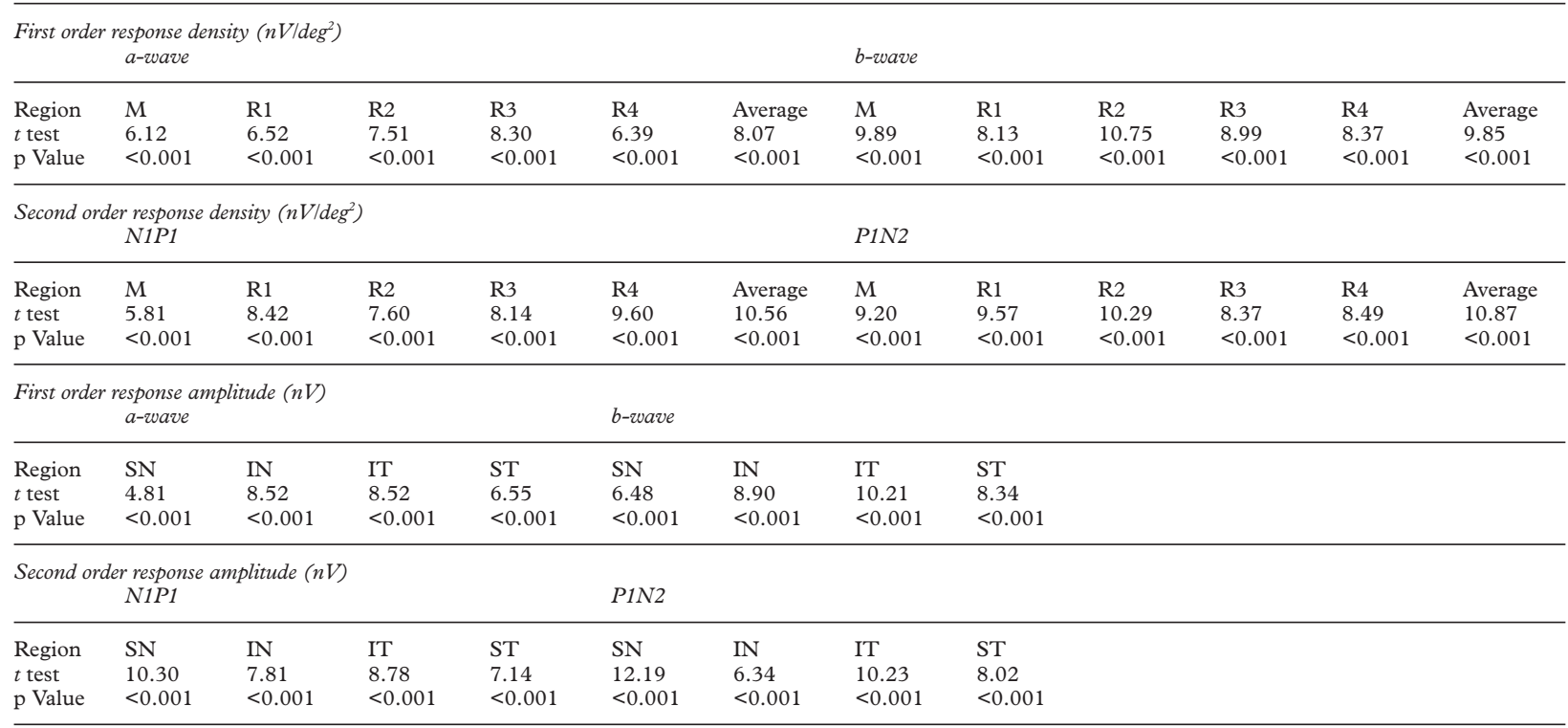


All procedures were approved by the ethics committee of the Hong Kong Polytechnic University.

MERG RECORDING

The stimulus of the MERG was presented as an array of hexagons (Fig 1) using a video projector (Electrohome ECP2000), which was driven by a Macintosh IIci computer. Data were collected using an analogue to digital converter board (National Instruments, NBMIO-16H) in the Macintosh computer. The 61 hexagon stimulus pattern used was scaled with eccentricity to provide approximately equal signal amplitude at each location (Fig 1). The distance from the screen to the subject was $40 \mathrm{~cm}$ and all the subjects had refraction corrected for the viewing distance. The angle subtended by the entire stimulus was about $56^{\circ}$
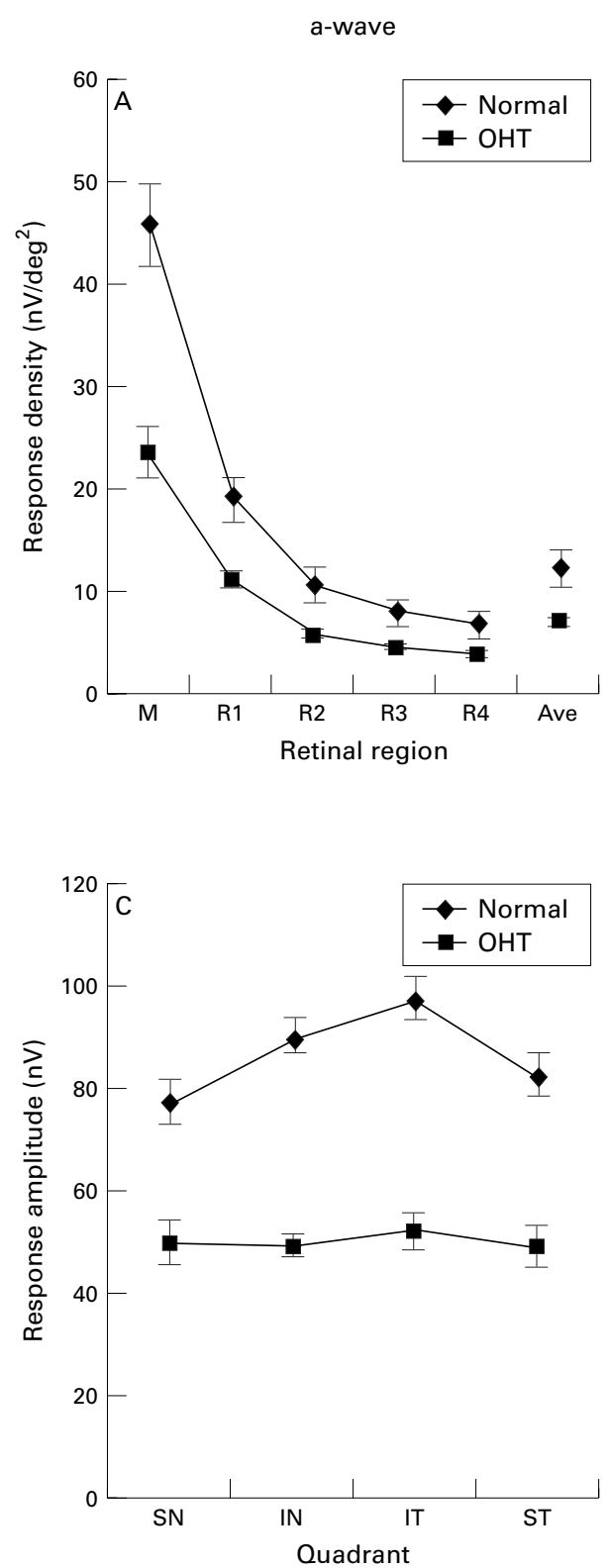

horizontally and vertically. The stimulus hexagons had luminances of $120 \mathrm{~cd} / \mathrm{m}^{2}$ (white) and $2 \mathrm{~cd} / \mathrm{m}^{2}$ (black); average luminance was about $61 \mathrm{~cd} / \mathrm{m}^{2}$ as measured with a Topcon BM-5 luminance meter, with $2^{\circ}$ field. The contrast of the stimuli was about $97 \%$.

The MERG system derives the impulse response to stimulation by cross correlating the stimulus sequence with the recorded ERG response. Each hexagon of the array was flickered according to a temporal binary $\mathrm{m}$ sequence $\left(2^{13}\right)$. The stimulus base interval chosen was $14.9 \mathrm{~ms}$ and the $\mathrm{m}$ sequence stimulation rate was the same as the frame rate of the stimulus monitor $(67 \mathrm{~Hz})$. The recording time for each stimulation cycle in this experiment was approximately 2 minutes. Each record was divided into eight slightly overlapping segments, and subjects were required to maintain
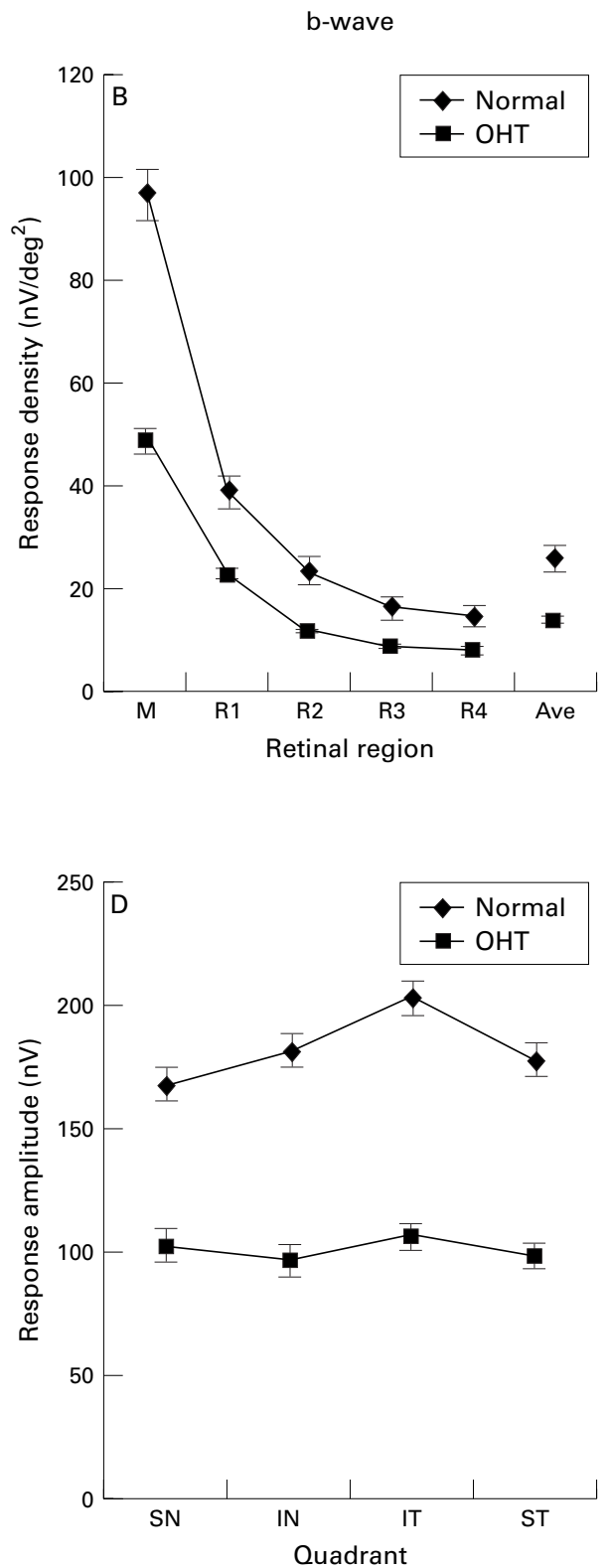

Figure 3 (A) Mean a-wave response densities, and (B) mean b-wave response densities of five ring grouping ( $M, R 1$, $R 2, R 3$, and R4) and average of all waveforms for OHT and control groups. (C) Mean a-wave response amplitude, and (D) mean b-wave response amplitude of four quadrant grouping ( $S N=$ superior-nasal, IN = inferior-nasal, $S T=$ superior-temporal, and IT = inferior-temporal). (Bars indicate SEM.) 
fixation without blinking for about 15 seconds for each recording segment. Four stimulus/ record files for averaging were obtained from each subject, and the total time taken for recording was about 8 minutes.

The responses to the hexagonal stimuli were averaged over five regions; the macula (central $3.5^{\circ}$ ), and concentric rings with eccentricities of: ring 1 (R1) $3.5^{\circ}-7^{\circ}$ : ring $2(\mathrm{R} 2) 7^{\circ}-13.5^{\circ}$ : ring 3 (R3) $13.5^{\circ}-20^{\circ}$ : ring 4 (R4) $20^{\circ}-28^{\circ}$ (Fig 1).

Dilated pupils were used in recording the ERG from both the control and the OHT subjects. One drop of tropicamide (1.0\%) (Alcon) was instilled in the examined eye and pupils were at least $7 \mathrm{~mm}$ in diameter during ERG recording. Dawson-Trick-Litzkow (DTL) electrodes were used for ERG recording. The amplifiers used were Grass P511K, with band pass from $1-100 \mathrm{~Hz}$, and gain of 100000 . A red fixation cross was presented at the centre of the display. Subjects were asked to maintain fixation at the cross, without blinking, during each recording segment. Subjects were rested between the recording segments. Any blinks or small eye movement during the recording seg-
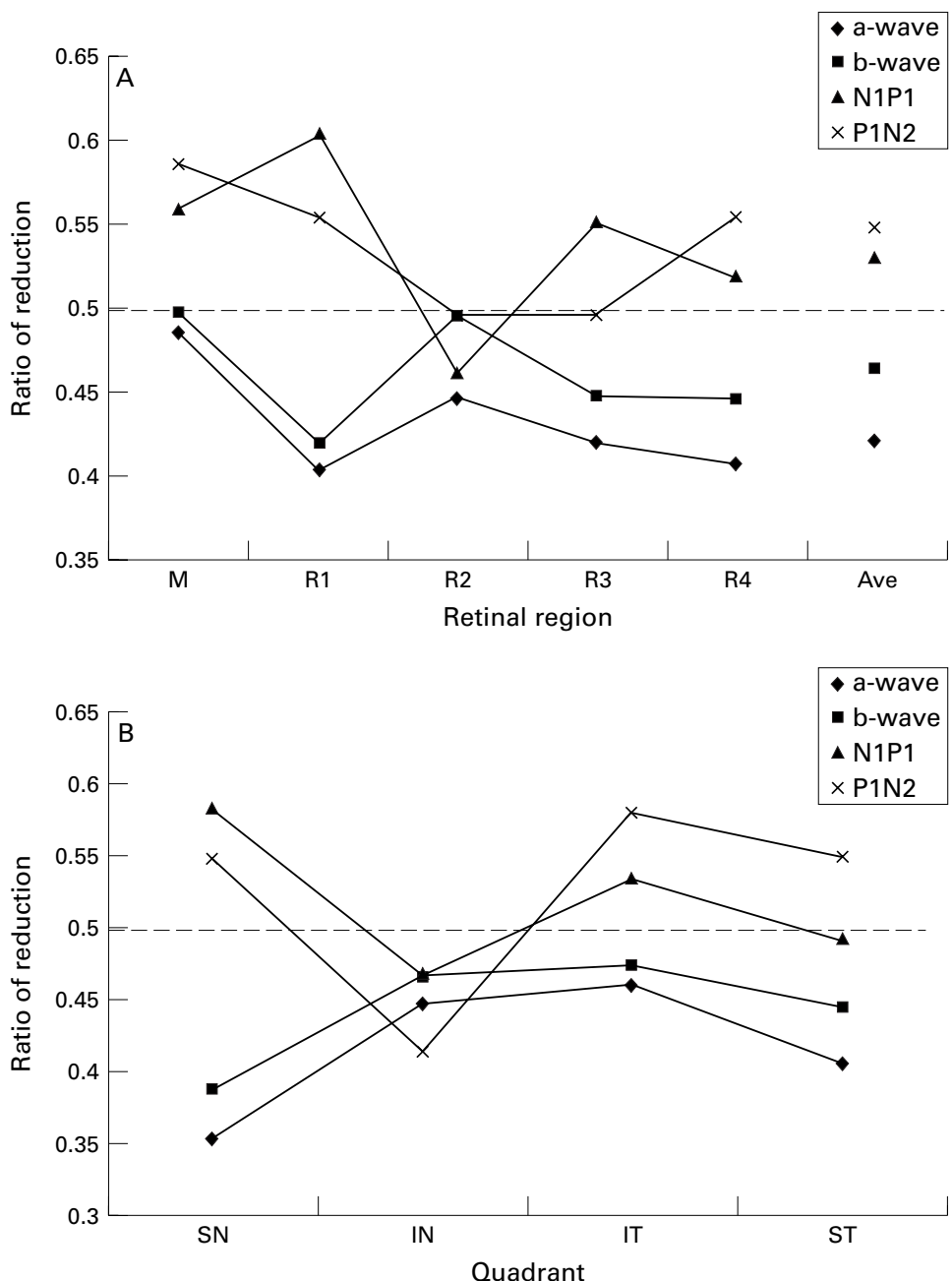

Figure 4 Ratio of the ERG response reduction in the OHT subjects compared with control subjects. (A) The ratios of $a$-wave, $b$-wave, N1P1, and P1N2 response densities in the five ring grouping. (B) The ratios of a-wave, $b$-wave, N1P1, and $P 1 N 2$ response amplitudes in the four quadrant grouping. ments caused ERG artefacts, and such segments were rejected and immediately recorded again.

\section{ANALYSIS}

The amplitude of the a-wave and b-wave in the first order kernel response, the amplitude from the first trough (N1) to the highest peak (P1) and the amplitude from the highest peak (P1) to the second trough $(\mathrm{N} 2)$ in the first slice of the second order kernel response were measured (Fig 2). The response density for the parameter at each ring of the stimulus was calculated; unpaired $t$ tests (assuming unequal variance) were used to compare the response changes in OHT and control subjects (five ring analysis).

There is a regional characteristic to glaucomatous degeneration; hence a comparison between the response in different retinal quadrants was performed. The MERG results for each eye were divided into four quadrants, superior-nasal (SN), superior-temporal (ST), inferior-nasal (IN) and inferior-temporal (IT) (Fig 1) to assess regional changes of the MERG response in OHT. Unpaired $t$ tests (assuming unequal variance) was used for statistical analysis (four quadrant analysis).

In addition, the ratios of the response reduction of the OHT group in both five ring and four quadrant analyses were calculated. The values of the ratio show the degree of the response reduction in both first and second order kernel analyses. The ratio of response reduction was defined as how much the response magnitude reduced in OHT (if the OHT value was non-recordable, the ratio equals 1 and the response is totally reduced; if the OHT value equals the normal value, the ratio equals 0 and no response reduction is shown).

1 - (OHT values/normal values)

Although it appears that ANOVA could be used for analysis of data at different eccentricities, these data are not really independent, so the more conservative approach of using multiple $t$ tests was chosen in the study.

\section{Results}

FIRST ORDER KERNEL RESPONSE

In the five ring analysis, the response densities of both a-wave and b-wave were significantly reduced in the OHT subjects $(\mathrm{p}<0.001$, Table 2, Fig 3A and B). The OHT subjects showed consistent reduction in mean response density at the macula and in the peripheral retina. In the four quadrant analysis, there are reductions in both a-wave and b-wave amplitude in the OHT subjects in all quadrants (Fig $3 \mathrm{C}$ and D). There was no quadrantal reduction of the response amplitude. These reductions in mean response amplitude in all quadrants are statistically significant $(\mathrm{p}<0.001$, Table 2$)$.

The ratio of response reduction $(1-(\mathrm{OHT})$ normal)) was calculated to examine the degree of loss for each parameter. In general, the response densities of a-wave and b-wave from the OHT patients were nearly half of those of the controls (Fig 4). The reduction ratios of the a-wave and b-wave in all examined regions 
were similar. It appeared that the OHT subjects had a general depression of the retinal responses in first order kernel analysis.

SECOND ORDER KERNEL RESPONSE

In the five ring analysis, both the response densities in N1P1 and P1N2 components were reduced in the OHT group in both macula and peripheral retina (Fig 5A and $\mathrm{B}$ ). These reductions in all regions were statistically significant ( $\mathrm{p}<0.001$, Table 2). There was a comparatively larger reduction in response density in the central retina (macula and ring 1) in OHT had than in the periphery (from ring 2 to ring 4) (Fig 4)

In the four quadrant analysis, there are reductions in the ERG amplitude of both $\mathrm{N} 1 \mathrm{P} 1$ and P1N2 components in the OHT subjects in all quadrants (Fig 5C and D).

N1P1
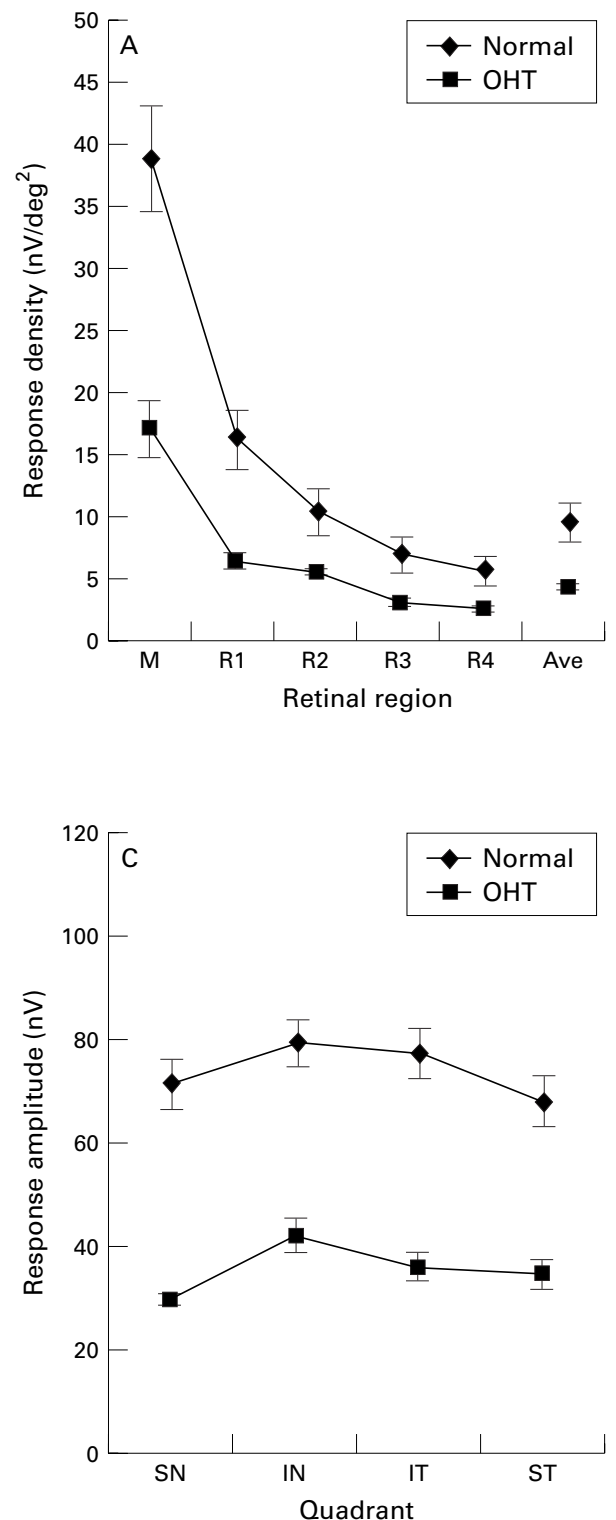

These reductions in mean response amplitude in all quadrants were statistically significant $(\mathrm{p}$ $<0.001$, Table 2). The inferior-nasal quadrant showed a smaller reduction of the response than the other three quadrants.

The OHT subjects showed a depression of the retinal response in the second order kernel, with response values less than half those of the controls (Fig 4). The reduction ratio values for $\mathrm{N} 1 \mathrm{P} 1$ and $\mathrm{P} 1 \mathrm{~N} 2$ indicate that the central retina (macula and ring 1 ) is more affected in OHT than is the periphery.

The second order kernel responses gave larger reduction ratios than those from first order kernel responses in both grouping methods (five ring and four quadrant). In Figure 4A and $\mathrm{B}$, the broken line marks the 0.5 ratio of reduction. The ratios of the first order kernel responses were, in general, below the line;
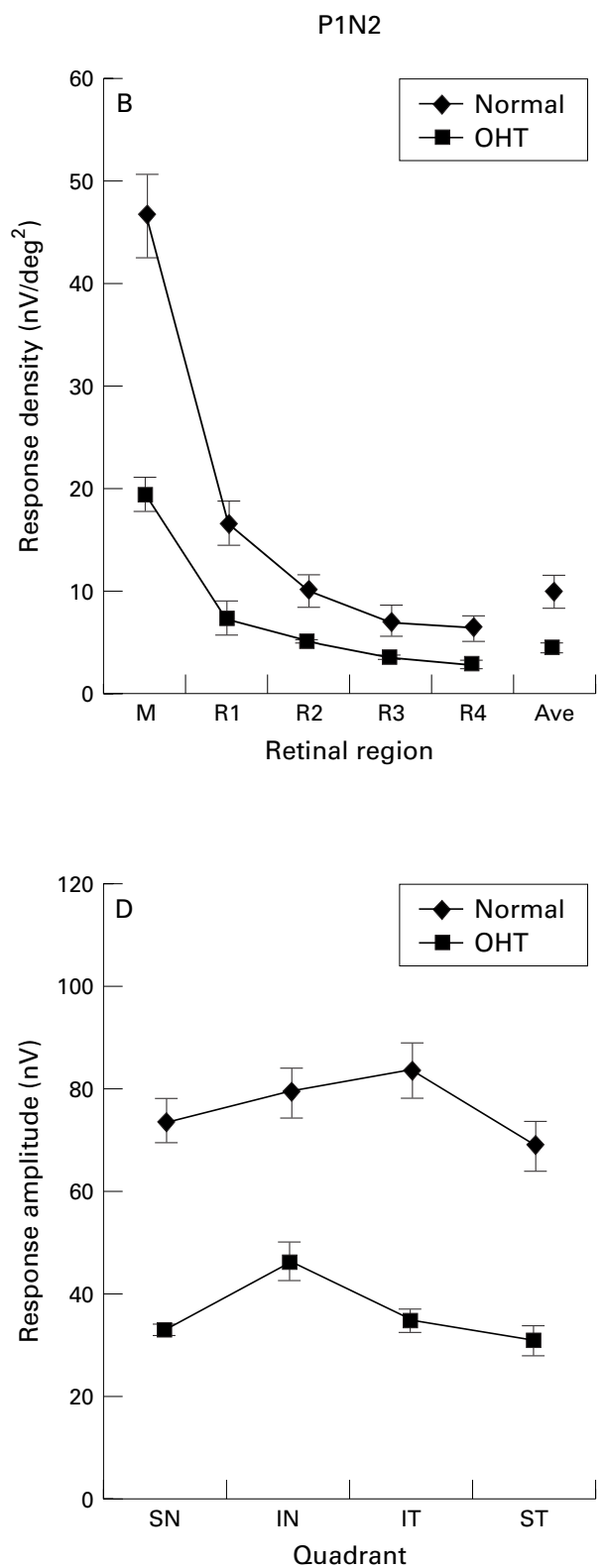

Figure 5 (A) Mean N1P1 response densities, and (B) mean P1N2 response densities of five ring grouping $(M, R 1, R 2$, $R 3$, and R4) and average of all waveforms for $O H T$ and control groups. (C) Mean N1P1 response amplitude, and (D) mean P1N2 response amplitude of four quadrant grouping ( $S N=$ superior-nasal, $I N=$ inferior-nasal, $S T=$ superior-temporal, and IT = inferior-temporal). (Bars indicate SEM.) 
A
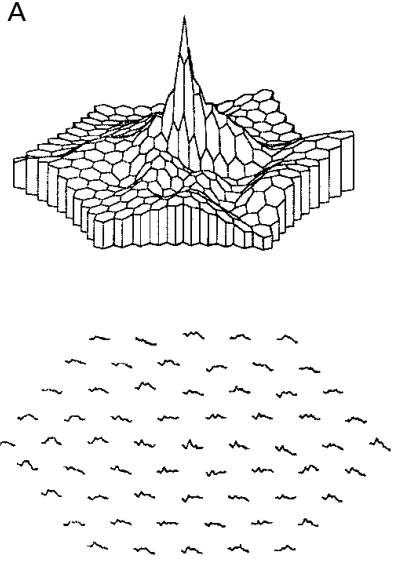

B

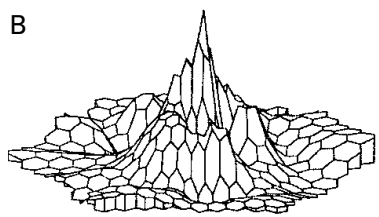

$-\cdots-\infty$

$\sim-\infty m-m$

$\sim m \sim m \sim m$

$-\infty-m-\infty n$

- $\cdots+\infty-\infty$

$\sim m \sim \infty$

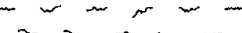
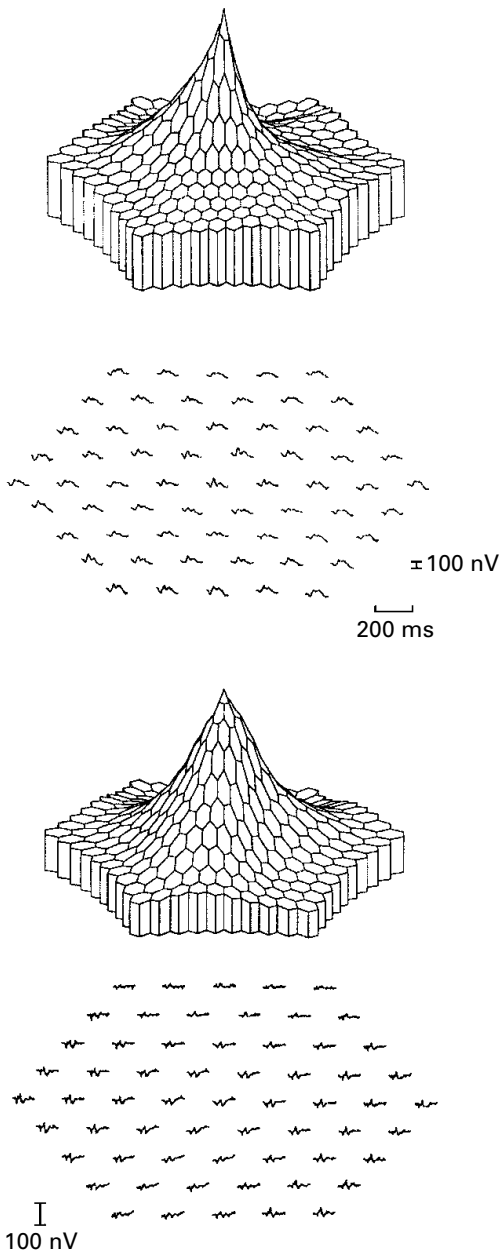

Figure 6 (A) Left: the general MERG (first order responses) reduction in one of the OHT subjects. Right: the MERG (first order response) in one of the normal subjects. (B) Left: the general MERG (second order responses) reduction in one of the OHT subjects. Right: the MERG (second order response) in one of the normal subjects.

while the ratios of the second order kernel were close to or above the line. The difference between the ratios of the first order kernels and those of the second order kernels were statistically significant (five ring analysis: $t=-5.09$, $\mathrm{p}<0.001$; four quadrant analysis: $t=-3.54$, $\mathrm{p}<0.01)$.

\section{Discussion}

First order kernel responses probably originate mainly in the outer retinal layer and partly in the inner retinal layer. ${ }^{12182021}$ The first order kernel response is similar to the response from the flash ERG. ${ }^{21}$ The second order kernel response probably originates mainly in the inner retinal layers and partly in the outer retinal layer. ${ }^{15} 1920$ Recent studies have found that the second order kernel response is reduced in conditions such as optic nerve atrophy, ${ }^{1520}$ glaucoma, ${ }^{15} 1822$ and diabetic retinopathy ${ }^{19}$ which damage the inner retina. This provides the rationale for the use of kernel analysis to assess OHT subjects who may have dysfunction of the inner retina.

In the present study, both first order and second order kernel responses showed reduction in the OHT subjects (Fig 6). The second order kernel response derived here is a non-linear response, which contains contributions from the inner retina. ${ }^{19}$ The inner retina is expected to be affected by early glaucomatous changes. ${ }^{23-28}$ The early retinal changes shown in the second order kernel response in OHT subjects in this study probably reflect damage of the inner retina. However, in this study, both the first and second order kernel responses were reduced in OHT subjects. The outer retinal components are probably involved in glaucomatous changes, and such changes are reflected in changes in the first order kernel. ${ }^{29-31}$

The contribution from inner retina in the first order kernel may be related to the frame rate of the stimulus. Fast and slow frame rates produce different characteristics in the response. ${ }^{21} 32$ The first order response in the fast frame rate condition (the condition used in this study) may include a relative contribution of non-linear overlapping effects which relate largely to activity of the inner retina..$^{18}{ }^{13}$ The second order response in the fast frame rate condition should be more related to activities of the inner retina. Thus, we saw a greater reduction in the response amplitude in the second order than in the first order kernel. However, the usefulness of the second order kernel in early glaucomatous changes under the slow frame rate condition is not yet clear. The frame rate used may be an important parameter in MERG assessment of eye disease.

In this study, there was a generalised reduction of the first order and second order kernel responses in OHT. The macula and ring 1 showed a comparatively large reduction in responses in the second order kernel. None of the OHT subjects in this study had any visual field defect and their measured thresholds were within the normal range (as calculated by the STATPAC in the Humphrey visual field analyser). This indicates that the MERG can detect retinal changes in OHT. Usually the early visual field defect in glaucoma is found in the paracentral area of Bjerrum $\left(10^{\circ}-20^{\circ}\right)$. It is believed that the first glaucomatous changes regions should lie in this area. However, in this study, no defect was found in visual field analysis and only general reduction in MERG was noticed in the examined area. The failure in detection of the small visual field changes may be because normal receptive fields in early glaucoma overlap with affected receptive fields in very early glaucoma. Scotomas may remain undetected until the affected region is large enough to be measured by visual field testing. ${ }^{31}$ However, the MERG is a direct measure of retinal function and the overlapping effect may not influence the measurement to such a degree.

The PERG is well established as detecting activity from the macula. The MERG showed a relative reduction in macular response and this is accord with the results of PERG measurements. Our results also agree with those of Bearse et $a l^{17}$ who found that the central retinal response in the second order kernel was reduced in glaucoma suspects. In addition, reduced macular function in glaucoma has also been shown by psychophysical measurements, such as colour vision and contrast 
sensitivity. ${ }^{34-37}$ General depression of the first order kernel was also reported by Vaegan and Buckland. ${ }^{38}$ They suggested the uniform reduction in all examined regions was consistent with a model of poor choriocapillary perfusion or microcirculatory loop closures. Reduced central retinal response in OHT also agrees with our previous study in assessing glaucoma. $^{22}$

The result implies that macular function is compromised in OHT and it may be a useful parameter for assessing early glaucomatous changes. In addition, the overall depression of the first order kernel might show how retinal function is affected by early glaucomatous changes. It is recommended the MERG can be used as a supplementary test for glaucoma suspects. The MERG cannot substitute for visual field testing, but it can give additional information in diagnosis of ocular hypertension. Both first and second order kernel analyses should be considered in cases of early glaucomatous changes. The patient with ocular hypertension may be considered to be in the high risk group of glaucoma suspects if the ratio of reduction of the second order kernel exceeds 0.5 (compared with control values), especially at the macula.

This study was supported by a research grant (UGC 0340/941) from the University Grant Council of Hong Kong.

1 Lewis TL, Fingeret M. Primary care of glaucomas. London: Appleton and Lange, 1993.

2 Quigley HA. Early detection of glaucomatous damage. II. Quigley HA. Early detection of glaucomatous damage. II.
Changes in the appearance of the optic disc. Surv OphthalChanges in the appea
mol 1985;30:111-26.

3 Graham PA. Epidemiology of primary glaucoma. In: Cairns JE, ed. Glaucoma. London: Grune and Stratton, 1986;1:56.

4 Trick GL. Pattern reversal retinal potentials in ocular hypertensives at high and low risk of developing glaucoma. Doc Ophthalmol 1987;65:79-85.

5 Weinstein GW, Arden GB, Hitchings RA, et al. The pattern electroretinogram (PERG) in ocular hypertension. Arch Ophthalmol 1988;106:923-31.

6 Nesher R, Trick GL. The pattern electroretinogram in retinal and optic nerve disease. A quantitative comparison of the pattern of visual dysfunction. Doc Ophthalmol 1991;77: $225-35$.

7 Pfeiffer N, Bach M, Tillmon B. Predictive value of pattern electroretinogram for development of visual field loss in ocular hypertensive patients. Invest Ophthalmol Vis Sci ocular hypertensive

8 Boschi A, Detry-Morel M, de Tourtchaninoff M, et al. Contribution of pattern electroretinogram and pattern visual tribution of pattern electroretinogram and pattern visual
evoked potential in early diagnosis of primary open angle evoked potential in early diagnosis of primary open ang
glaucoma. Bull Soc Belge Ophtalmol 1992;244:85-96.

glaucoma. Bull Soc Belge Ophtalmol 1992;244:85-96.
9 Pfeiffer N, Bach M. The pattern-electroretinogram in glaucoma and ocular hypertension. A cross-sectional and longitudinal study. Ger f Ophthalmol 1992;1:35-40.

10 O'Donaghue E, Arden GB, O'Sullivan F, et al. The pattern electroretinogram in glaucoma and ocular hypertension. $B$ f Ophthalmol 1992;76:387-94

11 Arai $\mathrm{M}$, Yoshimura $\mathrm{N}$, Sakane $\mathrm{H}$, et al. A 3-year follow-up study of ocular hypertension by pattern electroretinogram. Ophthalmologica 1993;207:187-95.
12 Sutter EE, Tran D. The field topography of ERG components in man-I. The photopic luminance response. Vis Res 1992;32:433-46.

13 Sutter EE. A deterministic time-domain nonlinear analysis. In: Pinter RB, Nabet B, eds. Nonlinear vision. London: CRC Press, 1992:171-220.

14 Chan HL, Brown B. Investigation of retinitis pigmentosa using the multifocal electroretinogram. Ophthalmic Physiol Opt 1998; 18:335-50.

15 Bearse Jr MA, Sutter EE, Smith DN, et al. Ganglion cell components of the human multifocal ERG are abnormal in optic nerve atrophy and glaucoma. Invest Ophthalmol Vis Sci 1995;36(suppl):S444.

16 Sasoh M, Yoshida S, Kuze M, et al. The multifocal electroretinogram in retinal detachment. Doc Ophthalmol 1998;94:239-52.

17 Sutter EE, Bearse Jr MA. Extraction of a ganglion cell component from the corneal response. In: Vision Science and its application. OSA Technical Digest Series. Washington DC: Optical Society of America 1995;1:310-3.

18 Bearse Jr MA, Sim D, Sutter EE, et al. Application of the multi-focal ERG to glaucoma. Invest Ophthalmol Vis Sci 1996;37(suppl):S511.

19 Palmowski AM, Sutter EE, Bearse Jr MA, et al. Mapping of retinal function in diabetic retinopathy using the multifocal electroretinogram. Invest Ophthalmol Vis Sci 1997;38:258696.

20 Bearse Jr MA, Sutter EE. Imaging localized retinal dysfunction with the multifocal electroretinogram. F Opt Soc Am (A) 1996;13:634-40.

21 Hood DC, Seiple W, Holopigian K, et al. A comparison of the components of the multi-focal and full-field ERGs. Vis Neurosci 1997;14:533-44.

22 Chan HL, Brown B. Multifocal ERG changes in glaucoma. Ophthal Physiol Opt 1999;19:306-16.

23 Quigley HA, Dunkelberger JR, Green WR. Chronic human glaucoma causes selectively greater loss of large optic nerve fibers. Ophthalmology 1988;95:357-63.

24 Arden GB, O'Sullivan F. Longitudinal follow up of glaucoma suspects tested with pattern electroretinogram. Bull Soc Belge Ophtalmol 1992;244:147-54.

25 Bach M, Funk J. Pattern electroretinogram and computerized optic nerve-head analysis in glaucoma suspects. Ger $\mathcal{F}$ Ophthalmol 1993;2:178-81.

26 Pfeiffer N, Tillmon B, Bach M. Predictive value of the pattern electroretinogram in high-risk ocular hypertension. Invest Ophthalmol Vis Sci 1993;34:1710-5.

27 Trick GL. Visual dysfunction in normotensive glaucoma. Doc Ophthalmol 1993;85:125-34.

28 Fernandez-Tirado FJ, Ucles P, Pablo L, et al. Electrophysiological methods in early glaucoma detection. Acta Ophthalmol 1994;72:168-74.

29 Sawada A, Nakazaki S, Nao-i N, Nagatomo A, Arai M. Retinal topography using multifocal electroretinogram in open-angle glaucoma. Invest Ophthalmol Vis Sci 1996; 37(suppl):S512

30 Bearse Jr MA, Sim D, Sutter EE, et al. Application of the multi-focal ERG to glaucoma. Invest Ophthalmol Vis Sci 1996;37(suppl):S511.

31 Vaegan, Buckland L. The retinal distribution of multifocal pattern and flash electroretinogram losses in glaucoma. Invest Ophthalmol Vis Sci 1996;37(suppl):S510.

32 Kondo M, Miyake Y, Horiguchi M, et al. Recording multifocal electroretinogram on and off responses in humans. Invest Ophthalmol Vis Sci 1998;39:574-80.

33 Sutter EE, Vaegan. Lateral interaction component and local luminance nonlinearities in the human pattern reversal ERG. Vis Res 1990;30:659-71.

34 Johnson CA. Selective versus non-selective losses in glaucoma. F Glaucoma 1994;3:32-44.

35 Trick GL. Visual impairment in glaucoma and ocular hypertension (OHT): results of a 4-year prospective study. Invest Ophthalmol Vis Sci 1991;32(suppl):1192.

36 Lachenmayr BJ, Drance SM. Central function and visual field damage in glaucoma. Int Ophthalmol 1992;16:203-9.

37 Ross JE. Clinical detection of abnormalities in central vision in chronic simple glaucoma using contrast sensitivity. Int Ophthalmol 1985;8:167-77.

38 Vaegan, Buckland L. The spatial distribution of ERG losses across the posterior pole of glaucomatous eyes in multifocal recordings. Aust NZ F Opthalmol 1996:24(2suppl):28-31. 